nephron

Practice
Nephron 2015;131:107-112

DOI: 10.1159/000439144
Received: June 5, 2015

Accepted: August 2, 2015

Published online: September 2, 2015

\title{
Choice of Reference Serum Creatinine in Defining Acute Kidney Injury
}

\author{
Edward D. Siew ${ }^{a-c, g} \quad$ Michael E. Matheny ${ }^{b-g}$ \\ ${ }^{a}$ Vanderbilt University Medical Center, Department of Medicine, Division of Nephrology and Hypertension, \\ ${ }^{b}$ Vanderbilt Center for Kidney Diseases (VCKD), ' GRECC, TVHS VA Medical Center, Veteran's Health Administration, \\ ${ }^{\mathrm{d}}$ Vanderbilt University Medical Center, Department of Biostatistics, ${ }^{e}$ Vanderbilt University Medical Center, \\ Department of Biomedical Informatics, ${ }^{f}$ Vanderbilt University Medical Center, Division of General Internal Medicine, \\ and ${ }^{9}$ Vanderbilt University Center for Population Health Informatics, Nashville, USA
}

\section{Key Words}

Acute kidney injury · Reference creatinine · Baseline creatinine

\begin{abstract}
Background/Aims: The study of acute kidney injury (AKI) has expanded with the increasing availability of electronic health records and the use of standardized definitions. Understanding the impact of AKI between settings is limited by heterogeneity in the selection of reference creatinine to anchor the definition of AKI. In this mini-review, we discuss different approaches used to select reference creatinine and their relative merits and limitations. Methods: We reviewed the literature to obtain representative examples of published baseline creatinine definitions when pre-hospital data were not available, as well as literature evaluating the estimation of baseline renal function, using PubMed and reference backtracing within known works. Results: (1) Pre-hospital creatinine values are useful in determining reference creatinine, and in high-risk populations, the mean outpatient serum creatinine value 7-365 days before hospitalization closely approximates nephrology adjudication, (2) in patients without pre-hospital data, the eGFR 75 approach does not reliably estimate true AKI incidence in most at-risk populations, (3) using the lowest inpatient serum creatinine may be reasonable, especially in those with preserved kidney function,
\end{abstract}

but may generously estimate AKI incidence and severity and miss community-acquired AKI that does not fully resolve, (4) using more specific definitions of AKI (e.g., KIDGO stages 2 and 3) may help to reduce the effects of misclassification when using surrogate values and (5) leveraging available clinical data may help refine the estimate of reference creatinine. Conclusions: Choosing reference creatinine for AKI calculation is important for AKI classification and study interpretation. We recommend obtaining data on pre-hospital kidney function, wherever possible. In studies where surrogate estimates are used, transparency in how they are applied and discussion that informs the reader of potential biases should be provided. Further work to refine the estimation of reference creatinine is needed. @ 2015 S. Karger AG, Basel

\section{Introduction}

Modern epidemiologic studies of acute kidney injury (AKI) have been facilitated by the increasing availability of electronic health records $[1,2]$. The latter has enabled

Contribution from the AKI \& CRRT 2015 Symposium at the 20th International Conference on Advances in Critical Care Nephrology, Manchester Grand Hyatt, San Diego, Calif., USA, February 17-20, 2015.

\section{KARGER 125}

(c) 2015 S. Karger AG, Base

$1660-8151 / 15 / 1312-0107 \$ 39.50 / 0$

E-Mail karger@karger.com

www.karger.com/nef
Dr. Edward D. Siew

Department of Medicine, Division of Nephrology and Hypertension

Vanderbilt University Medical Center, 116121 st Avenue South

MCN S-3223, Nashville, TN 37232-2372 (USA)

E-Mail edward.siew@vanderbilt.edu 
Table 1. Different approaches used to selecting reference creatinine values when pre-hospital data were not available

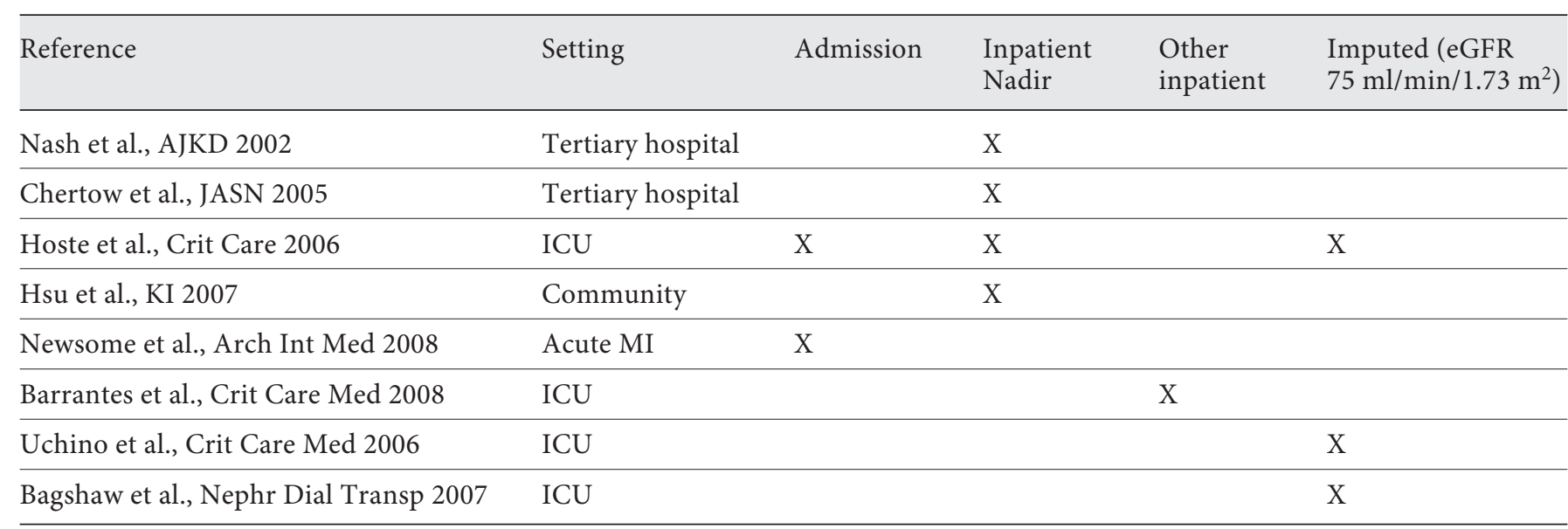

Table 2. Evolution of consensus recommendations for choosing reference creatinine

\begin{tabular}{llll}
\hline Criteria & RIFLE [5] & AKIN [6] & KDIGO [7] \\
\hline Date & 2004 & 2007 & 2012 \\
\hline Reference & $\begin{array}{l}\text { Not specifically defined. If not available, } \\
\text { back-calculate a serum creatinine using an eGFR } \\
\text { of } 75 \mathrm{ml} / \mathrm{min} / 1.73 \mathrm{~m}^{2} \text { using the MDRD equation }\end{array}$ & $\begin{array}{l}\text { Rolling 48 h } \\
\text { window }\end{array}$ & $\begin{array}{l}\text { Not specifically defined. If not available, use } \\
\text { lowest serum creatinine during hospitalization }\end{array}$ \\
\hline
\end{tabular}

investigators to leverage laboratory data to study this disease with greater granularity and accuracy by reducing reliance on administrative codes. An important challenge that has emerged is choosing a suitable reference creatinine value to anchor the change in serum creatinine used to determine criteria fulfillment. Ideally, the value chosen accurately reflects steady-state kidney function prior to the onset of AKI. However, information on pre-hospital kidney function is often partially or entirely missing, prompting the use of surrogate reference values that can influence the quantification of AKI and its severity, comparisons of this disease between settings and the longterm study of this disease. In this mini-review, we highlight how different approaches to choosing reference creatinine can impact the ascertainment of AKI, affect understanding of its association with outcomes and discuss potential strategies toward choosing appropriate reference serum creatinine. We identified articles through a PubMed search for AKI and observational cohort studies, and also reviewed the references in the articles that were found and in previously known articles for relevance and inclusion.

\section{The Impact of Choice of Reference Creatinine in AKI Studies}

A variety of approaches have been used to define reference creatinine (table 1). Differences in these approaches limit the ability to make accurate comparisons between observational cohorts, are accompanied by varying degrees of AKI misclassification that also alter estimates of prognosis and association with other covariates (e.g., novel biomarkers) [3, 4]. Common approaches include using admission creatinine, the lowest inpatient creatinine, other inpatient or outpatient surrogates or imputing an arbitrary value. The original RIFLE criteria did not specify how to choose reference creatinine, but recommended the imputation of a value calculated from an estimated glomerular filtration rate of $75 \mathrm{ml} / \mathrm{min} / 1.73 \mathrm{~m}^{2}$ (eGFR 75 approach) when missing [5]. Iterative definitions suggest alternative approaches including using a rolling 48-hour window to reduce the need for pre-hospitalization value (AKIN) or the lowest inpatient serum creatinine when missing (KDIGO; table 2) [6, 7]. 


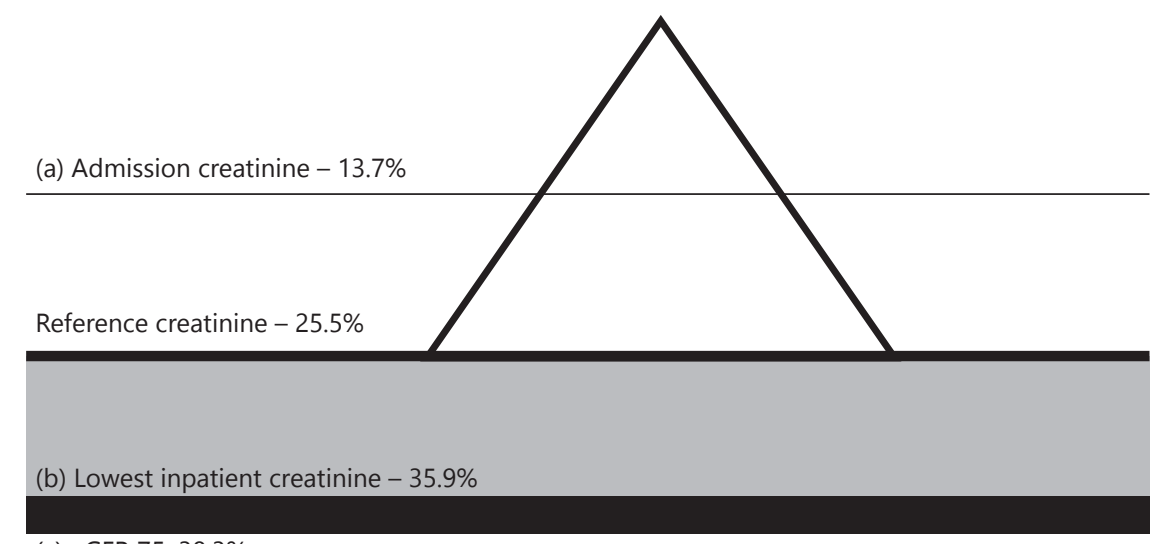

(c) eGFR $75-38.3 \%$

Fig. 1. Potential direction of bias associated with surrogate estimates of reference serum creatinine. The actual burden of AKI reflected by a mountain analogy (actual incidence 25.5\%). Using admission creatinine (a) will be sensitive to AKI that either only worsens after admission or occurs following hospitalization. As a result, it may only see the 'tip' of the mountain (observed incidence $13.7 \%$ ), tend to underestimate severity and prognosis will seem worse for a given stage than is actually the case. Using the lowest inpatient creatinine (b) may overestimate AKI to some degree

In a study consisting of 4,863 hospitalized adults [3], the magnitude and direction of AKI misclassification were examined by comparing these approaches to selecting reference creatinine using the most recent outpatient serum creatinine 7-365 days prior to admission as a gold standard. A summary of these findings is illustrated in figure 1 . In this study, the reference standard yielded an incidence AKI of 25.5\%. However, when the eGFR 75 approach was used, the observed incidence increased to $38.3 \%$. Most of this inflation was likely due to an erroneous assumption that patients with chronic kidney disease (CKD) had low-normal kidney function, resulting in a false-positive diagnosis of AKI. Conversely, using the admission serum creatinine value reduced the observed incidence of AKI to $13.7 \%$, likely due to poor sensitivity of community-acquired AKI. When the lowest inpatient serum creatinine was used, the observed incidence of AKI also increased to a maximum of $35.9 \%$. Potential contributors for this include, but are not limited to, lower creatinine production in the midst of acute illness (e.g., true lowering of reference creatinine) or the effects of volume expansion (e.g., false lowering of reference creatinine) [8]. Using lowest inpatient serum creatinine does im-

Reference Serum Creatinine in Defining AKI (gray zone up to $35.9 \%$ ) depending on whether the lowering of creatinine is confounded by factors such as fluid overload or reflects true lowering due to reduced generation. Severity may also tend to be inflated, and the associated prognosis per given stage may seem lower than the one that was actually observed. Not observable is community-acquired AKI that does not begin to resolve. The eGFR 75 approach (c) will mistakenly assign patients with stable CKD as having AKI, overestimate AKI incidence (38.3\%) and underestimate prognosis.

prove sensitivity to community-acquired AKI (relative to admission creatinine) unless it does not resolve following admission. Notably, most of the misclassification observed for all of these approaches was observed in the mildest stages of injury (i.e., RIFLE risk/AKIN or KDIGO stage 1).

This study also examined the potential impact of AKI staging and short-term mortality. As using the eGFR 75 approach tended to assign an overly optimistic estimate of reference creatinine, this approach also tended to overestimate the stage of severity. Consequently, the shortterm mortality associated with AKI tended to be lower than the one that was actually observed. The direction of bias observed was similar when using the minimum inpatient serum creatinine. However, the magnitude of bias in using the minimum inpatient value may be reduced in some cases where there is a true lowering of serum creatinine that precedes the AKI (e.g., decreased creatinine production). The degree to which this is observable depends on knowing the pre-morbid kidney function. In contrast, use of first admission serum creatinine tends to underestimate AKI severity as it will be insensitive to community-acquired AKI. As a result, the mortality associated with a given AKI stage would tend to be higher 
than observed when using a pre-admission reference standard.

In summary, different surrogate approaches for choosing reference serum creatinine in AKI studies can cause bidirectional misclassification of AKI status, its severity and examination of associated outcomes. The effects are most pronounced with mild stages of injury and when these surrogates are applied to a large proportion of the study population. These findings highlight the need to interpret study results accordingly, and the need to obtain information on pre-morbid kidney function whenever possible.

\section{Defining Reference Serum Creatinine When Pre-Hospitalization Data Are Available}

Because of the dynamic variability of serum creatinine during acute illness, obtaining information on pre-admission kidney function can be informative. The complexity in the timing, frequency and setting in which serum creatinine is ordered prior to hospitalization favors the use of clinical adjudication to determine an appropriate reference value; however, its application in large data sets is usually not feasible.

One study attempted to determine an algorithm that might approximate clinical adjudication by examining pre-hospital kidney function in the 2 years prior to index hospitalization among high-risk patients with evidence of AKI or abnormally high serum creatinine during hospitalization [9]. An adjudicated reference standard was determined by a panel of nephrologists and agreement was compared to the following: (1) the most recent outpatient serum creatinine, (2) the mean outpatient serum creatinine, (3) the most recent in/outpatient serum creatinine and (4) the lowest outpatient serum creatinine within 7-365 days prior to admission. The effects of varying the time window for value inclusion were also explored for 7-730 and 1-730 days. The intra-class correlation coefficient (ICC) was found to be highest for the mean outpatient (ICC 0.91, 95\% CI 0.88-0.92) approach. Rates of AKI misclassification using a $0.3 \mathrm{mg} / \mathrm{dl}$ threshold were estimated to be $13.7 \%$ using the most recent outpatient value, $11.1 \%$ for mean outpatient, $12.1 \%$ for most recent in/outpatient and $20.3 \%$ for the lowest outpatient value. The overall direction of misclassification and their potential explanations varied according to approach. For example, the lowest outpatient value tended to be lower than the adjudicated value, possibly due to progression of underlying kidney disease, interim AKI or possible lab error. In contrast, the most recent values tended to be slightly higher than the adjudicated values in some cases, potentially due to capture of early community-acquired AKI. Notably, including inpatient values (most recent in/ outpatient) did allow for more patients to be incorporated (i.e., only those with inpatient values in the preceding 7-365 days).

Expanding the window to 7-730 days prior to admission allowed for more patients to be included but did decrease agreement with the adjudicated reference standard, particularly when using the lowest outpatient value. Including values from the week prior to admission tended to decrease agreement for mean and most recent approaches, likely due to the effects of acute illness.

In summary, in this selected high-risk population, the mean outpatient value 7-365 days most closely approximated clinical adjudication in choosing a pre-hospital reference creatinine. Differences in estimated AKI misclassification between mean and most recent outpatient and most recent in/outpatient values, however, were small and use of most recent in/outpatient did reduce selection bias. The lowest outpatient values taken over the course of a year performed less well, likely due to the effects of progressive CKD or interim AKI. Extending algorithms beyond a year prior to hospitalization or inclusion of values within a week prior to hospitalization may reduce selection bias but do so at the expense of accuracy.

\section{Defining Reference Serum Creatinine When Pre-Hospitalization Data Are Not Available}

A critical challenge in choosing reference creatinine is that pre-admission serum creatinine data is often missing. The likelihood of missingness is usually nonrandom and associated with numerous patient-related (e.g., age, comorbidity, distance from hospital) and hospital-related (tertiary vs. primary, presence of an EHR) factors. For these reasons, investigators often have to choose between selection bias that result from excluding patients without pre-hospital creatinine data and the risk of misclassification that result from using surrogate values.

In the absence of pre-hospitalization data, one common approach has been eGFR 75 approach. This method assumes a relatively healthy population with low rates of $\mathrm{CKD}$, and thus, the resulting magnitude of misclassification is directly proportional to the amount of CKD in the 
cohort. Another more insidious limitation of this approach is that using a single value will underestimate the distribution of reference serum creatinine within the population the more it is used. This will yield conservative standard error rates regarding the association of AKI with other covariates that do not reflect the actual uncertainty in the data [10].

Another approach has been using the minimum serum creatinine during hospitalization. The rationale for this approach is that it provides a reasonable estimate of reference creatinine among patients without AKI and among those with AKI who recover and may also be helpful in examining hospital-acquired AKI. In some cases, the lowest inpatient serum creatinine may even more accurately reflect kidney function. For example, in prolonged critical illness or sepsis $[8,11]$, decreased creatinine generation may result in lower serum creatinine values. However, other studies have suggested the presence of other confounders, such as fluid overload, that can impact AKI diagnosis [12]. This can result from either the masking of changes in serum creatinine or lowering of artificial lowering of the reference standard.

These limitations suggest potential benefit in more sophisticated approaches to estimate reference creatinine. One commonly used method that uses known patient characteristics and accounts for uncertainty in the multiple estimations of missing values is known as multiple imputation $[10,13]$. In one study, use of multiple imputation to estimate reference creatinine was more accurate than the eGFR 75 approach, primarily by increasing specificity and positive predictive value among those with lower eGFR values [14]. The utility of this approach likely depends on the degree of missing data and the quality of the data that is available.

\section{Summary}

The choice of reference creatinine for AKI calculation has important implications for study interpretation. The following are the key issues to be aware of when choosing reference creatinine: (1) pre-hospital creatinine values can be useful in determining reference creatinine value, and in high-risk patient populations, the mean outpatient serum creatinine value 7-365 days prior to hospitalization closely approximates nephrology adjudication, (2) in patients without pre-hospital data, the eGFR 75 approach is unlikely to provide an accurate estimation of true AKI incidence in most patient popula- tions or to help understand the relationship between AKI and other clinical variables, (3) using the lowest inpatient serum creatinine may be reasonable, especially in those whose values indicate preserved kidney function, but may provide an overall generous estimate of AKI incidence and severity as well be less sensitive to community-acquired AKI that does not fully resolve, (4) in studies with larger proportions of patients with missing preadmission data, using more specific definitions of AKI (e.g., KIDGO stages 2 and 3 ) and focusing less on interstage differences may help to reduce the effects of misclassification and (5) using available high quality data may help refine the estimate of reference creatinine in those with missing data. Techniques leveraging these data, such as multiple imputation, can be superior to complete case analysis, even at higher levels of missingness in some cases.

In summary, we recommend that whenever possible, effort be taken to obtain data on pre-hospital kidney function. However, restricting studies to only those where such 'ideal' determination of reference creatinine value is available is not feasible as it may introduce bias and limit progress. The use of surrogate estimates in retrospective or prospective observational studies is not unreasonable when pre-hospital baseline is not available. However, when applied, transparency in how they are applied and discussion that explicitly informs the reader of potential biases and in which direction of likely bias should be provided. This is especially important in long-term studies of kidney function following AKI, where accurate assessment of pre-AKI kidney function is paramount. We also recommend caution in attempting to use surrogate estimates to guide clinical decisionmaking or trial enrollment on an individual-patient level, especially if interventions that are being considered carry significant risk. Leveraging available high-quality data to estimate reference creatinine can help reduce bias. However, future work to define the optimal application of these techniques and continued refinements in the approach of determining reference creatinine are needed.

\section{Disclosure Statement}

E.D.S. is supported by the Vanderbilt Nephrology Divisional Funds, NIH DK92192-07, and Veterans Health Administration HSR\&D IIR-073-3 and does not report any conflicts related to the enclosed work. M.E.M. is supported by grants Veterans Health Administration HSR\&D IIR-11-292 and IIR-13-052 and does not have any conflicts to report. 


\section{References}

1 Go AS, Parikh CR, Ikizler TA, Coca S, Siew ED, Chinchilli VM, Hsu CY, Garg AX, Zappitelli M, Liu KD, Reeves WB, Ghahramani N, Devarajan P, Faulkner GB, Tan TC, Kimmel PL, Eggers P, Stokes JB; Assessment Serial Evaluation, and Subsequent Sequelae of Acute Kidney Injury Study Investigators: The assessment, serial evaluation, and subsequent sequelae of acute kidney injury (ASSESSAKI) study: design and methods. BMC Nephrol 2010;11:22.

2 Parikh CR, Coca SG, Thiessen-Philbrook H, Shlipak MG, Koyner JL, Wang Z, Edelstein CL, Devarajan P, Patel UD, Zappitelli M, Krawczeski CD, Passik CS, Swaminathan M, Garg AX; TRIBE-AKI Consortium: Postoperative biomarkers predict acute kidney injury and poor outcomes after adult cardiac surgery. J Am Soc Nephrol 2011;22:17481757.

3 Siew ED, Matheny ME, Ikizler TA, Lewis JB, Miller RA, Waitman LR, Go AS, Parikh CR, Peterson JF: Commonly used surrogates for baseline renal function affect the classification and prognosis of acute kidney injury. Kidney Int 2010;77:536-542.

4 Bagshaw SM, Uchino S, Cruz D, Bellomo R, Morimatsu H, Morgera S, Schetz M, Tan I, Bouman C, Macedo E, Gibney N, Tolwani A, Oudemans-van Straaten HM, Ronco C, Kellum JA; Beginning and Ending Supportive Therapy for the Kidney (BEST Kidney) Investigators: A comparison of observed versus es- timated baseline creatinine for determination of RIFLE class in patients with acute kidney injury. Nephrol Dial Transplant 2009;24: 2739-2744.

5 Bellomo R, Ronco C, Kellum JA, Mehta RL, Palevsky P; Acute Dialysis Quality Initiative Workgroup: Acute renal failure - definition, outcome measures, animal models, fluid therapy and information technology needs: the second international consensus conference of the acute dialysis quality initiative (ADQI) group. Crit Care 2004;8:R204-R212.

6 Mehta RL, Kellum JA, Shah SV, Molitoris BA, Ronco C, Warnock DG, Levin A; Acute Kidney Injury Network: Acute kidney injury network: report of an initiative to improve outcomes in acute kidney injury. Crit Care 2007; 11:R31.

7 Kellum JA, Lameire N; KDIGO AKI Guideline Work Group: Diagnosis, evaluation, and management of acute kidney injury: a KDIGO summary (Part 1). Crit Care 2013;17:204.

8 Doi K, Yuen PS, Eisner C, Hu X, Leelahavanichkul A, Schnermann J, Star RA: Reduced production of creatinine limits its use as marker of kidney injury in sepsis. J Am Soc Nephrol 2009;20:1217-1221.

9 Siew ED, Ikizler TA, Matheny ME, Shi Y, Schildcrout JS, Danciu I, Dwyer JP, Srichai M, Hung AM, Smith JP, Peterson JF: Estimating baseline kidney function in hospitalized patients with impaired kidney function. Clin J Am Soc Nephrol 2012;7:712-719.
10 Donders AR, van der Heijden GJ, Stijnen T, Moons KG: Review: a gentle introduction to imputation of missing values. J Clin Epidemiol 2006;59:1087-1091.

11 Prowle JR, Kolic I, Purdell-Lewis J, Taylor R, Pearse RM, Kirwan CJ: Serum creatinine changes associated with critical illness and detection of persistent renal dysfunction after AKI. Clin J Am Soc Nephrol 2014;9:10151023.

12 Liu KD, Thompson BT, Ancukiewicz M, Steingrub JS, Douglas IS, Matthay MA, Wright P, Peterson MW, Rock P, Hyzy RC, Anzueto A, Truwit JD; National Institutes of Health National Heart, Lung, and Blood Institute Acute Respiratory Distress Syndrome Network: Acute kidney injury in patients with acute lung injury: impact of fluid accumulation on classification of acute kidney injury and associated outcomes. Crit Care Med 2011;39:2665-2671.

13 van der Heijden GJ, Donders AR, Stijnen T, Moons KG: Imputation of missing values is superior to complete case analysis and the missing-indicator method in multivariable diagnostic research: a clinical example. J Clin Epidemiol 2006;59:1102-1109.

14 Siew ED, Peterson JF, Eden SK, Moons KG, Ikizler TA, Matheny ME: Use of multiple imputation method to improve estimation of missing baseline serum creatinine in acute kidney injury research. Clin J Am Soc Nephrol 2012;8:10-18. 